\title{
Can caregivers report their care recipients' post-stroke hospitalizations and outpatient visits accurately? Findings of an Asian prospective stroke cohort
}

Shilpa Tyagi', Gerald Choon-Huat Koh ${ }^{1 *}$, Nan Luo ${ }^{1}$, Kelvin Bryan Tan², Helen Hoenig ${ }^{3}$, David B. Matchar ${ }^{4}$, Joanne Yoong ${ }^{1}$, Eric A. Finkelstein ${ }^{4}$, Kim En Lee ${ }^{5}$, N. Venketasubramanian ${ }^{6}$, Edward Menon ${ }^{7}$, Kin Ming Chan ${ }^{8}$, Deidre Anne De Silva ${ }^{9}$, Philip Yap ${ }^{10}$, Boon Yeow Tan ${ }^{11}$, Effie Chew ${ }^{12}$, Sherry H. Young ${ }^{13}$, Yee Sien $\mathrm{Ng}^{14}$, Tian Ming Tu ${ }^{15}$, Yan Hoon Ang ${ }^{10}$, Keng He Kong ${ }^{16}$, Rajinder Singh ${ }^{15}$, Reshma A. Merchant ${ }^{17}$, Hui Meng Chang ${ }^{9}$, Tseng Tsai Yeo ${ }^{18}$, Chou Ning ${ }^{18}$, Angela Cheong ${ }^{1}$, Yu Li Ng${ }^{2}$ and Chuen Seng Tan ${ }^{1}$

\begin{abstract}
Background: Health services research aimed at understanding service use and improving resource allocation often relies on collecting subjectively reported or proxy-reported healthcare service utilization (HSU) data. It is important to know the discrepancies in such self or proxy reports, as they have significant financial and policy implications. In high-dependency populations, such as stroke survivors, with varying levels of cognitive impairment and dysphasia, caregivers are often potential sources of stroke survivors' HSU information. Most of the work conducted on agreement analysis to date has focused on validating different sources of self-reported data, with few studies exploring the validity of caregiver-reported data. Addressing this gap, our study aimed to quantify the agreement across the caregiverreported and national claims-based HSU of stroke patients.
\end{abstract}

Methods: A prospective study comprising multi-ethnic stroke patient and caregiver dyads $(N=485)$ in Singapore was the basis of the current analysis, which used linked national claims records. Caregiver-reported health services data were collected via face-to-face and telephone interviews, and similar health services data were extracted from the national claims records. The main outcome variable was the modified intraclass correlation coefficient (ICC), which provided the level of agreement across both data sources. We further identified the amount of over- or under-reporting by caregivers across different service types.

Results: We observed variations in agreement for different health services, with agreement across caregiver reports and national claims records being the highest for outpatient visits (specialist and primary care), followed by hospitalizations and emergency department visits. Interestingly, caregivers over-reported hospitalizations by approximately $49 \%$ and under-reported specialist and primary care visits by approximately 20 to 30\%.

(Continued on next page)

\footnotetext{
* Correspondence: gerald_koh@nuhs.edu.sg

${ }^{1}$ Saw Swee Hock School of Public Health, National University of Singapore,

12 Science Drive 2, \#10-01, Singapore 117549, Singapore

Full list of author information is available at the end of the article
}

(c) The Author(s). 2018 Open Access This article is distributed under the terms of the Creative Commons Attribution 4.0 International License (http://creativecommons.org/licenses/by/4.0/), which permits unrestricted use, distribution, and reproduction in any medium, provided you give appropriate credit to the original author(s) and the source, provide a link to the Creative Commons license, and indicate if changes were made. The Creative Commons Public Domain Dedication waiver (http://creativecommons.org/publicdomain/zero/1.0/) applies to the data made available in this article, unless otherwise stated. 


\begin{abstract}
(Continued from previous page)
Conclusions: The accuracy of the caregiver-reported HSU of stroke patients varies across different service types. Relatively more objective data sources, such as national claims records, should be considered as a first choice for quantifying health care usage before considering caregiver-reported usage. Caregiver-reported outpatient service use was relatively more accurate than inpatient service use over shorter recall periods. Therefore, in situations where objective data sources are limited, caregiver-reported outpatient information can be considered for low volumes of healthcare consumption, using an appropriate correction to account for potential under-reporting.
\end{abstract}

Keywords: Healthcare, Validation, Caregiver, Intraclass correlation coefficient, Stroke

\section{Background}

Agreement or repeatability is the degree of concordance across two or more data sources (or raters) for the variables studied. Gisev et al. [1] described inter-rater agreement (IRA) as "the extent to which different raters assign the same precise value for each item being rated" [1]. Context is important in conducting and reporting agreement studies, as the accuracy of the results is affected by the population characteristics, recall period, type and frequency of utilization and mode of data collection [2].

Stroke survivors are high utilizers of healthcare resources, and studies have tried to quantify the cost of stroke for resource allocation and policy purposes. Such studies often rely on self-reported usage [3-7]. Moreover, stroke as an illness is unique, as it leads to multiple disabilities and varying levels of cognitive impairment, with survivors often depending on a caregiver for different needs, including keeping track of healthcare service utilization. Health services research aimed at understanding service use and improving resource allocation often relies on collecting subjectively reported healthcare service utilization data. With an ageing population, the dependence on caregivers to collect care recipient-related information will significantly increase. Thus, it is important to assess the validity of the healthcare service utilization of stroke patients reported by their caregivers. Most of the work performed on agreement to date has focused on the agreement between different sources of self-reported data, with few exploring the validity of proxy-reported data compared with electronic records [8-11]. Moreover, none of these studies were based on proxy reporters of stroke survivors. The current study will address the abovementioned knowledge gaps.

In addition to a dearth of research on the validity of proxy-reported data for stroke, there is a general methodological issue regarding how data in such studies are treated. Typically, healthcare utilization agreement studies have attempted to either quantify agreement based on traditional measures, such as Cohen's kappa or the intraclass correlation coefficient (ICC) [8, 10, 12-18], or used a two-step process to extend the analysis to describing the reporting patterns of utilizers [11, 19-26]. Studies often end up dichotomizing or categorizing their healthcare service utilization data (e.g., counts or discrete events), which could potentially result in a loss of power. Addressing this issue, we will consider an approach whereby we will quantify the agreement based on original count data.

\section{Methods}

The primary aim of the current research was to investigate the absolute agreement between caregiver-reported and national claims record-based healthcare service utilization in stroke patients in an Asian setting. The objectives of the study were the following: (i) to report the absolute agreement between the two data sources in the number of healthcare visits post-stroke for inpatient, emergency department (ED), specialist outpatient clinic (SOC) and primary care (PC) services and (ii) to quantify over- or under-reporting by caregivers across different healthcare services utilized by stroke patients, with national claims records taken as the gold standard.

Researchers have previously reported several weaknesses of administrative databases in conducting research, such as limited information on the clinical care delivered, varying accuracy across different data elements, limited information on reasons for utilization or the severity of illness and limited accuracy in identifying clinical diagnoses or procedures [27]. Therefore, few considerations should be considered while assuming an administrative database, such as a claims data record, as a gold standard in research. First, whether the purpose of the research aligns with the purpose of maintaining the administrative database should be considered. The national claims record in Singapore maintains an islandwide database of acute and outpatient healthcare service utilization and associated expenditures. Current research is focused on studying the reporting pattern for healthcare service utilization, requiring us to extract utilization information from the national claims record. Because the information extracted constitutes the primary data fields captured in the national claims record, we are better positioned to assume it as a gold standard with respect to the data fields considered. However, if we were to use an administrative database to diagnose a disease condition, we would be relying on the International 
Classification of Diseases (ICD) codes or procedural coding within the administrative database, which are inherently plagued with accuracy issues arising due to variations in coding at the physician level and the limited specificity of certain diseases. In this scenario, consideration of an administrative database as a gold standard may not be feasible unless validation studies provide evidence of the accuracy of the information captured [28, 29].

Another consideration regarding healthcare service utilization information is the coverage of services under the administrative database. The national claims record covers inpatient healthcare utilization across both the public and private sectors, while the outpatient healthcare coverage is limited mainly to the public sector or government-run SOCs and polyclinics. Therefore, we are relatively more confident in considering the national claims record as a gold standard for inpatient services than for outpatient services. To elaborate on potential implications, we will be more certain about the over- or under-reporting of caregiver-reported inpatient services, whereas we must interpret outpatient services based on our findings. If we encounter over-reporting of outpatient services by caregivers, we will not be able to comment on whether there is true over-reporting or if it is an artefact due to the limited coverage of outpatient services in the national claims record. However, if our findings suggest the under-reporting of outpatient services by caregivers, we will be more certain of the finding, with the magnitude of under-reporting being a conservative estimate of the true value.

We hypothesized the following: (i) the absolute agreement to be higher for inpatient service use and lower for $\mathrm{ED}, \mathrm{SOC}$ and PC service use; and (ii) the inpatient service utilization to be over-reported and the $\mathrm{ED}$, SOC and PC service utilization to be under-reported. Our hypotheses are based on previously reported findings, whereby salient or more serious events such as hospitalizations are reported with greater accuracy than are non-salient or less serious events such as outpatient visits [2]. Moreover, proxies over-report hospitalizations and tend to under-report outpatient service use $[8,30]$.

\section{Singapore stroke study (S3)}

Stroke survivors and caregivers were recruited from all five tertiary hospitals in Singapore from December 2010 to September 2013 under the Singapore Stroke Study (S3). Eligibility criteria included the following: Singaporean or permanent residents, older than 40 years old and residing in Singapore for 1 year of follow-up, stroke was clinically confirmed with imaging evidence, recent diagnosis (symptoms occurring no more than 4 weeks prior to seeking care) and the absence of global aphasia. Caregivers were immediate or extended family members or friends, older than 21 years of age, the main people providing care and taking responsibility for the patient and not fully paid for caregiving.

Caregiver-reported stroke patients' healthcare service utilization (counts of hospitalizations, ED visits, SOC visits and $\mathrm{PC}$ visits) in the past 3 months at each interview over 1 year was considered in the current analysis. The 3-month recall period was determined based on the past literature supporting the association of shorter recall periods with a greater accuracy of reporting utilization [2]. Information across both S3 and the national claims record was matched based on the quarterly availability of healthcare usage data, with data availability ranging from one quarter to all four quarters post-index stroke. We summed the utilization reported across all available 3-month periods or quarters for both data sources, which implies that if caregiver X-reported and national claims record-based healthcare service utilization information is available over the first post-stroke quarter (0-3 months) and second quarter (3-6 months), utilization over these two quarters will be summed, and the absolute agreement for caregiver $\mathrm{X}$ will be calculated based on this summed utilization. This quarter-based approach was taken because the caregiver-reported information was captured over each quarter with a consistent recall period of 3 months, and it enabled us to maximize the sample size available for the current analysis.

\section{Analysis}

Agreement analysis is based on quantifying how well two or more data sources (or raters) report concordance on a common phenomenon being studied. This phenomenon in the current study is healthcare service utilization, captured as count variables. Our two data sources are caregivers of stroke survivors (proxy reported) and the national claims record. Two of the commonly used agreement measures are Cohen's kappa for binary or categorical variables and the ICC for continuous variables. This traditional ICC, commonly used to quantify agreement for continuous data [31,32], is based on analysis of variance (ANOVA) [33]. Expanding on this approach, we introduce the concept of modified ICC, which can be defined as the proportion of betweengroup variance by total variation (including both betweenand within-group variance) and can be used to quantify agreement for both continuous and count data $[34,35]$.

$$
\text { Modified ICC }=\frac{\text { between-group variance }}{\text { between-group variance }+ \text { within-group variance }}
$$

Here, between-group variance is a measure of heterogeneity in the utilization pattern of stroke survivors and within-group variance is a result of deviation from the absolute agreement between healthcare service utilization 
information for a stroke survivor from both data sources. In simple terms, if there is high absolute agreement between both caregiver-reported and national claims record-based utilization information, the within-group variance (in the denominator) would be lower, resulting in a higher value of the modified ICC. For more details regarding the calculation of the modified ICC, refer to Additional file 1.

Adopting a generalized linear mixed model (GLMM)based approach, agreement for healthcare service utilization between caregiver-reported and national claims record data can be quantified as this modified ICC, with applicability extending to count or discrete event variables. The GLMM approach provides flexibility to not only deal with non-Gaussian variables but also enable the incorporation of random and fixed effects into the model [36]. We incorporated a random intercept for each stroke survivor and a fixed term denoting the data source $(0=$ national claims record; $1=$ caregiver report) to obtain between-group variance and withingroup variance, respectively (Model 1). We ran four models in total, one for each service studied, assuming a Poisson distribution for the total visits with log-link. The GLMM approach provided a modified ICC on the original scale (i.e., total number of visits) and the latent scale (i.e., log-transformed scale), and we reported both scales for completeness. We used the bootstrap approach to obtain the $95 \%$ confidence interval for the modified ICC estimates (both latent and original scales). From Model 1, the exponentiated value of the coefficient for the data source variable, where national claims data was the reference, indicated the over- or under-reporting of healthcare service utilization by the caregiver (i.e., incidence rate ratio). We also reported the traditional ICC for the number of visits by assuming the total number of visits was Gaussian-distributed. R software version 3.3.3 was used in the analysis [37], with the rptR package used to compute the modified ICC [35]. A $P$-value less than 0.05 was set as the threshold for statistical significance.

\section{Results}

A total of 485 stroke patient-caregiver dyads were available for the current analysis after matching across both databases and excluding those dyads with patient deaths occurring within the observation period of 12 months $(n=37)$.

The baseline socio-demographic characteristics of the stroke survivors are provided in Table 1 . The majority of the stroke survivors were less than 65 years of age, Chinese, married, religious, and male. Almost all stroke survivors were admitted to a subsidized ward class for index-stroke episodes, with approximately $8 \%$ opting for non-subsidized wards. The proportions of patients with mild, moderate and severe stroke, as measured by the National Institutes of Health Stroke Scale (NIHSS), were $58 \%, 36 \%$ and $6 \%$, respectively. Approximately $45 \%$ of the patients were moderately to severely dependent, as measured by the Barthel Index at baseline, with approximately $38 \%$ having slight or no dependence and $17 \%$ having complete dependence. More than half of the stroke patients had no cognitive impairment at baseline. The average age of caregivers at baseline was 47 years. More than half of them were spousal caregivers, followed by adult-child, siblings and others (distant relatives and friends) as caregivers. The majority were Chinese females, and approximately three-quarters were married. Slightly more than one-third of the caregivers were providing care to multiple care recipients, and approximately three-fourths were co-residing with the stroke patient.

As shown in Table 2, the caregivers reported outpatient service utilization by stroke patients with greater accuracy than inpatient service utilization. The highest agreement was observed for the SOC service, with a modified ICC value of 0.64 (95\% CI: $0.56,0.69)$, and this was closely followed by the PC service, with the agreement measured as a modified ICC of 0.61 (95\% CI: 0.52, 0.66). Within the inpatient services, the volume of hospitalizations was reported with greater accuracy than was the volume of ED services consumed, with an ICC between a caregiver-reported and claims record-based volume of hospitalizations across both data sources of 0.48 (95\% CI: 0.41, 0.55). The lowest agreement was observed for ED services, with a modified ICC value of 0.39 (95\% CI: 0.08, 0.49).

All the services utilized by stroke patients were under-reported by their caregivers, except for the number of hospitalizations, which was over-reported. Caregivers reported $49 \%$ more hospitalizations than those found in the national claims record (95\% CI: 1.22, 1.82). The magnitude of under-reporting by caregivers, compared with utilization in the national claims record, varied across different services, with under-reporting ranging from 72 to $87 \%, 23$ to $35 \%$ and 9 to $28 \%$ for ED, SOC and PC services, respectively (Table 2). We conducted additional sensitivity analyses to determine whether the reported results changed with the addition of relevant covariates. In general, our adjusted analysis results were similar to those reported here after taking into account the variability attributable to the covariates, such as stroke survivors' socio-demographic, clinical and functional characteristics (refer to Additional file 2: Tables S1, S2 and S3 for details regarding the analyses and results).

\section{Discussion}

Our study demonstrates a discordance between proxy and national claims record reports of health care utilization 
Table 1 Socio-demographic and clinical characteristics of utilizers (stroke patients)

\begin{tabular}{|c|c|c|c|c|c|c|c|}
\hline Variable & & $\begin{array}{l}\text { Frequency/ } \\
\text { Mean }\end{array}$ & Percent/SD & Variable & & $\begin{array}{l}\text { Frequency/ } \\
\text { Mean }\end{array}$ & Percent/SD \\
\hline \multirow[t]{3}{*}{ Age } & & & & & Severe Cognitive & 53 & 13 \\
\hline & $<65$ years & 292 & 60 & & Impairment (1-17) & & \\
\hline & $\geq 65$ years & 193 & 40 & $\begin{array}{l}\text { Frontal Assessment } \\
\text { Battery }(F A B)^{a}\end{array}$ & & 14 & 4 \\
\hline \multirow[t]{3}{*}{ Gender } & & & & Centre for & & 6 & 5 \\
\hline & Male & 318 & 66 & Epidemiological & & & \\
\hline & Female & 167 & 34 & Scale (CESD) $)^{a}$ & & & \\
\hline \multirow[t]{3}{*}{ Ethnicity } & & & & Caregiver identity & Spouse & 259 & 54 \\
\hline & Chinese & 326 & 67 & & Adult-Child & 128 & 26 \\
\hline & Non-Chinese & 159 & 33 & & Sibling & 29 & 6 \\
\hline \multirow[t]{2}{*}{ Religion } & & & & & Others & 27 & 6 \\
\hline & Religion & 440 & 91 & & None & 40 & 8 \\
\hline
\end{tabular}

Marital status

$\begin{array}{lll}\text { Married } & 347 & 72 \\ \text { Single } & 138 & 28\end{array}$

Ward class

Unsubsidized

Subsidized

Stroke Type

$\begin{array}{lll}\text { Ischaemic } & 420 & 87 \\ \text { Non-ischaemic } & 62 & 13\end{array}$

National Institute of Health Stroke Scale (NIHSS)

\begin{tabular}{|c|c|}
\hline Mild (0-4) & 265 \\
\hline $\begin{array}{l}\text { Moderately Severe } \\
(5-14)\end{array}$ & 165 \\
\hline Severe (15-24) & \\
\hline
\end{tabular}

Barthel Index

$\begin{array}{lll}\begin{array}{l}\text { Independence (100) } \\ \text { Slight Dependence } \\ \text { (91-99) }\end{array} & 60 & 24 \\ \begin{array}{l}\text { Moderate } \\ \text { Dependence (61-90) }\end{array} & 128 & 30 \\ \begin{array}{l}\text { Severe Dependence } \\ \text { (21-60) }\end{array} & 65 & 15 \\ \begin{array}{l}\text { Total Dependence } \\ (0-20)\end{array} & 73 & 17 \\ & & \end{array}$

Modified Rankin Scale (mRS) $(0-2)$

Moderate or Severe 277 Disability (3-5)

Mini-Mental State

No Cognitive

Examination (MMSE)
Table 1 Socio-demographic and clinical characteristics of utilizers (stroke patients) (Continued)

${ }^{a}$ The mean and SD were reported for these variables

for stroke patients. Furthermore, we illustrate a novel approach for quantifying agreement across different data sources using the original data (e.g., counts or discrete events), without dichotomizing or categorizing our data. In the past, some studies limited their results to reporting single agreement measures such as kappa or the traditional ICC $[8,10,12-18]$, while others opted for a second analysis, reporting factors associated with over- or underreporting [11, 19-26]. Often with healthcare data, count variables are binarized to facilitate the use of kappa, compromising on the information available, which potentially results in a loss of power, and our study addressed this gap in the literature.

Previous studies have reported that more salient events, such as hospitalizations, are remembered with greater accuracy than are less salient events, such as outpatient visits [2, 11]. However, our results show caregiver-reported agreement to be higher for outpatient (SOC and PC) services compared with that for inpatient services. A possible explanation could be that past literature mainly focused on self-reported estimates, while our study is among the few to analyse caregiver-reported usage and, to the best of our knowledge, the first to do so in stroke populations. Another possible explanation could be the shorter recall period in our study, during which caregivers reported more frequent events with greater accuracy than did less frequent events. In the past, a shorter recall period was recommended to yield a more accurate reporting of frequent healthcare events [2]. Moreover, agreement estimates for salient events are higher for studies involving a longer recall period $[11,30,38]$ than a shorter recall period $[8,17]$. Another possibility could be that the construct of saliency may be operationalized differently for caregivers, with the frequency of healthcare events having greater significance

$$
\text { (24-30) }
$$

Mild Cognitive Impairment (18-23) 
Table 2 Summary statistics and agreement estimates for healthcare usage by stroke patients over 1 year post-stroke

\begin{tabular}{|c|c|c|c|c|c|c|}
\hline & $\begin{array}{l}\text { Caregiver-reported visits } \\
\text { Median ( } 25 \text { th }-75 \text { th) }\end{array}$ & $\begin{array}{l}\text { Objective administrative visits } \\
\text { Median ( } 25 \text { th - 75th) }\end{array}$ & $\begin{array}{l}\text { ICC } \\
\text { Estimate } \\
(95 \% \mathrm{Cl})\end{array}$ & $\begin{array}{l}\text { Modified ICC, } \\
\text { original scale } \\
\text { Estimate } \\
(95 \% \mathrm{Cl})\end{array}$ & $\begin{array}{l}\text { Modified ICC, } \\
\text { latent scale } \mathrm{b}^{\mathrm{b}} \\
\text { Estimate }(95 \% \mathrm{Cl})\end{array}$ & $\begin{array}{l}\text { Over- or under- } \\
\text { reporting effect } \\
\text { IRR }(95 \% \mathrm{Cl})\end{array}$ \\
\hline $\begin{array}{l}\text { Hospitalization } \\
\text { Mean (SD) }\end{array}$ & $\begin{array}{l}0(0-1) \\
0.55(0.89)\end{array}$ & $\begin{array}{l}0(0-0) \\
0.38(0.93)\end{array}$ & $0.48(0.41,0.55)$ & $0.55(0.35,0.63)$ & $0.54(0.42,0.61)$ & $1.49(1.22,1.82)$ \\
\hline $\begin{array}{l}\text { ED visits } \\
\text { Mean (SD) }\end{array}$ & $\begin{array}{l}0(0-0) \\
0.11(0.42)\end{array}$ & $\begin{array}{l}0(0-1) \\
0.53(1.38)\end{array}$ & $0.10(0.01,0.19)$ & $0.10(0.00,0.11)$ & $0.39(0.08,0.49)$ & $0.19(0.13,0.28)$ \\
\hline $\begin{array}{l}\text { SOC visits } \\
\text { Mean (SD) }\end{array}$ & $\begin{array}{l}2(0-4) \\
2.80(3.37)\end{array}$ & $\begin{array}{l}3(1-5) \\
3.87(4.31)\end{array}$ & $0.51(0.44,0.57)$ & $0.59(0.51,0.67)$ & $0.64(0.56,0.69)$ & $0.71(0.65,0.78)$ \\
\hline $\begin{array}{l}\text { Primary care visits } \\
\text { Mean (SD) }\end{array}$ & $\begin{array}{l}1(0-2) \\
1.50(1.96)\end{array}$ & $\begin{array}{l}1(0-3) \\
1.83(2.14)\end{array}$ & $0.47(0.40,0.53)$ & $0.60(0.49,0.69)$ & $0.61(0.52,0.66)$ & $0.81(0.72,0.91)$ \\
\hline
\end{tabular}

${ }^{\mathrm{a}} \mathrm{ICC}$ assumes the healthcare usage has a Gaussian distribution

${ }^{\mathrm{b}}$ Modified ICC assumes the healthcare usage has a Poisson distribution

than the saliency of the type of healthcare event. Similar findings were reported by a study involving proxy-reported healthcare use, with agreement for outpatient service use being higher than that for inpatient service use, with a Lin's concordance correlation coefficient of 0.67 and 0.55 , respectively [8]. Compared with the traditional ICC, higher estimates of the modified ICC were observed for all healthcare services. Therefore, treating count data as continuous may lead to the underestimation of the magnitude of agreement by approximately $15 \%, 16 \%$ and $28 \%$ for inpatient, SOC and PC services, respectively.

Assuming the national claims record as a reference, we observed the over-reporting of inpatient service use and under-reporting of outpatient service use by stroke patients according to their caregivers, which is in accordance with previous studies $[8,15,30]$. For example, for every 10 hospitalizations in the national claims database, caregivers reported 15 hospitalizations, whereas for every 10 PC visits, caregivers reported 8 visits. There are potential implications of these inaccuracies in reporting, whereby using caregiver-reported hospitalizations could inflate the usage by $50 \%$ and lead to the overestimation of usage and economic burden. This result is important because hospitalizations constitute a major proportion of total healthcare costs. Similarly, the use of caregiver-reported PC usage may yield conservative estimates, with a $20 \%$ lower usage; this may affect decisionmaking in terms of the allocation of resources to outpatient settings or evaluation of interventions targeted at improving outpatient service use post-stroke. Based on current findings, as much as possible, the use of administrative data sources (e.g., national claims record) for healthcare service utilization information is suggested. Depending on the availability and feasibility of acquiring administrative data, caregiver-reported estimates of outpatient service use can be considered for low volumes of consumption, taking into consideration 20 to $30 \%$ under-reporting by inflating the caregiver-reported utilization appropriately. When these caregiver-reported estimates of outpatient service usage are independent variables in the regression analysis, measurement error models can be used to avoid biased estimates. It is important to note that the decision to choose one of the data sources, proxy-reported or administrative databases (including national claims records), should not only be based on the accuracy of information but also consider factors such as feasibility, timeliness, availability, cost of acquisition, and comprehensiveness of the data fields collected.

Our study has some limitations. We assumed the national claims record to be the gold standard source of healthcare service utilization data, which may not be the case. Although inpatient service utilization in claims records includes both public and private sectors, PC service utilization may be limited to the public sector. However, the healthcare system in Singapore is based on a heavily subsidized public healthcare sector to enable affordability, which in turn incentivizes consumers to utilize healthcare resources in the public sector. Moreover, our findings of the under-reporting of $\mathrm{PC}$ usage are a conservative estimate of the actual under-reporting by the proxies, considering the limited coverage of PC in the national claims record. Because agreement estimates are context-dependent and vary across different populations, the generalizability of our results will be limited to the population of stroke survivors.

Our study has the following strengths. First, we adopted a modelling approach to calculate absolute agreement while accommodating for non-Gaussian healthcare data. Moreover, adopting this methodology allowed us to study both agreement measures and reporting patterns (overvs. under-reporting) within a single analysis rather than calculating them separately, as has been done in the past [11, 19-26, 39]. Second, to the best of our knowledge, we are among the first to report the agreement of caregivers or proxy-reported healthcare utilization with the national claims record (an objective data source). Recently, a few authors reported agreement for proxy-reported resource 
utilization [8-11]. However, none of these studies focused on stroke survivor populations, who are relatively high utilizers. The higher relevance of our study also lies in the fact that post-stroke survivors have speech impairment, cognitive impairment, and physical disabilities of varying magnitudes, resulting in a high reliance on caregivers to report the healthcare resource use of their care recipient. Moreover, with a rapidly ageing population globally, the relevance of caregiver-reported resource utilization will increase. Thus, it is crucial to determine the accuracy of their reporting patterns.

\section{Conclusion}

In conclusion, we described the agreement between caregiver-reported and national claims record-based healthcare utilization of stroke survivors by utilizing a model-based approach and reporting the modified ICC. Caregiver-reported outpatient service use is relatively more accurate than inpatient service use over shorter recall periods based on a comparison of modified ICC estimates. Therefore, objective data sources (such as a national claims record) should be considered for quantifying healthcare usage. Depending on the availability and feasibility of acquiring such administrative data, caregiver-reported estimates of outpatient service usage can be considered for low volumes of consumption. Furthermore, the modified ICC can be used for both Gaussian and Poisson data, making the modified ICC a universal agreement measure for continuous and count variables. Future research initiatives should expand the current analysis to study the agreement between caregiver-reported healthcare costs and explore adjusted agreement analysis with appropriate covariates.

\section{Additional files}

Additional file 1: Modified ICC calculation from generalized linear mixed-effects models.pdf. (PDF $99 \mathrm{~kb}$ )

Additional file 2: Supplementary tables of adjusted analysis.pdf. (PDF 113 kb)

\section{Abbreviations}

ANOVA: Analysis of variance; ED: Emergency department; GLMM: Generalized linear mixed model; ICC: Intraclass correlation coefficient; IRA: Inter-rater agreement; NIHSS: National Institute of Health Stroke Scale; PC: Primary care; S3: Singapore Stroke Study; SOC: Specialist outpatient clinic

\footnotetext{
Acknowledgements

We would like to thank the medical staff at the public tertiary hospitals for assisting with the recruitment of patients and their caregivers. We would also like to thank all the participants in our study for their participation and cooperation.
}

\section{Funding}

The Singapore Stroke Study was funded by a Health Services Research Competitive Research Grant from the National Medical Research Council, Singapore. The funders were not involved in the data collection, analysis or writing of this manuscript.
This research is supported by the Singapore Ministry of Health's National Medical Research Council under the Centre Grant Programme - Singapore Population Improvement Centre (NMRC/CG/C026/2017_NUHS).

\section{Availability of data and materials}

The datasets generated and/or analysed during the current study are not publicly available because the national claims data obtained from the Ministry of Health of Singapore (for the purpose of the current study) are not an openly available/accessible data source. The datasets are available from the corresponding author upon reasonable request.

\section{Authors' contributions}

ST, GCHK, NL, KBT and CST conceptualized and designed the current study. GCHK, LN, KBT, KEL, NV, EM, KMC, DADS, PY, BYT, EC, SHY, YSN, TMT, YHA, KHK, RS, RAM, HMC, TTY, CN, AC and YLN were involved in data acquisition. $\mathrm{HH}$ and DBM provided medical domain expertise and JY and EAF provided financial domain expertise to guide data acquisition. ST and CST conducted the analysis and interpretation of data. ST prepared the first draft of the manuscript and incorporated subsequent revisions. GCHK supervised the study. All authors read and approved the final manuscript.

\section{Ethics approval and consent to participate}

Ethical approval was provided by the National University of Singapore Institutional Review Board, SingHealth Centralized Institutional Review Board and the National Health Group Domain Specific Review Board. Written informed consent was collected from all participants in S3.

\section{Consent for publication}

Not applicable

\section{Competing interests}

The authors declare that they have no competing interests.

\section{Publisher's Note}

Springer Nature remains neutral with regard to jurisdictional claims in published maps and institutional affiliations.

\section{Author details}

'Saw Swee Hock School of Public Health, National University of Singapore, 12 Science Drive 2, \#10-01, Singapore 117549, Singapore. ${ }^{2}$ Policy Research \& Economics Office, Ministry of Health, Singapore, Singapore. ${ }^{3}$ Physical Medicine and Rehabilitation Service, Durham VA Medical Centre, Durham, USA. ${ }^{4}$ Program in Health Services and Systems Research, Duke-NUS Graduate Medical School, Singapore, Singapore. ${ }^{5}$ Lee Kim En Neurology Pte Ltd, Singapore, Singapore. ${ }^{6}$ Raffles Neuroscience Centre, Raffles Hospital, Singapore, Singapore. ${ }^{7}$ St. Andrew's Community Hospital, Singapore, Singapore. ${ }^{8}$ Mount Alvernia Hospital, Singapore, Singapore. ${ }^{9}$ National Neuroscience Institute, Singapore General Hospital campus, Singapore, Singapore. ${ }^{10}$ Geriatric Medicine, Khoo Teck Puat Hospital, Singapore, Singapore. ${ }^{11}$ St. Luke's Hospital, Singapore, Singapore. ${ }^{12}$ Department of Rehabilitation Medicine, National University Hospital, Singapore, Singapore. ${ }^{13}$ Department of Rehabilitation Medicine, Changi General Hospital, Singapore, Singapore. ${ }^{14}$ Department of Rehabilitation Medicine, Singapore General Hospital, Singapore, Singapore. ${ }^{15}$ Department of Neurology, National Neuroscience Institute, Neurology, Tan Tock Seng Hospital, Singapore, Singapore. ${ }^{16}$ Department of Rehabilitation Medicine, Tan Tock Seng Hospital, Singapore, Singapore. ${ }^{17}$ Department of Medicine, Yong Loo Lin School of Medicine, National University of Singapore, Singapore, Singapore.

${ }^{18}$ Department of Neurosurgery, National University Hospital, Singapore, Singapore.

Received: 5 June 2018 Accepted: 16 October 2018

Published online: 25 October 2018

\section{References}

1. Gisev N, Bell JS, Chen TF. Interrater agreement and interrater reliability: key concepts, approaches, and applications. Res Soc Adm Pharm. 2013;9(3):330-8.

2. Bhandari A, Wagner T. Self-reported utilization of health care services: improving measurement and accuracy. Med Care Res Rev. 2006;63(2): 217-35 
3. Asplund K, Ashburner S, Cargill K, Hux M, Lees K, Investigators MDftGI. Health care resource use and stroke outcome: multinational comparisons within the GAIN international trial. Int J Technol Assess Health Care. 2003; 19(2):267-77.

4. Christensen MC, Morris S. Association between disability measures and short-term health care costs following intracerebral hemorrhage. Neurocrit Care. 2008;9(3):313-8.

5. Dewey HM, Thrift AG, Mihalopoulos C, et al. Cost of stroke in Australia from a societal perspective. Stroke. 2001;32(10):2409-16.

6. Fattore $G$, Torbica A, Susi A, et al. The social and economic burden of stroke survivors in Italy: a prospective, incidence-based, multi-Centre cost of illness study. BMC Neurol. 2012;12(1):137.

7. Snozzi P, Blank PR, Stroke in Switzerland STD. Social determinants of treatment access and cost of illness. J Stroke Cerebrovasc Dis. 2014; 23(5):926-32.

8. Lee TA, Fuhlbrigge AL, Sullivan SD, et al. Agreement between caregiver reported healthcare utilization and administrative data for children with asthma. J Asthma. 2007:44(3):189-94.

9. Wehby GL, Jones MP, Ullrich F, Lou Y, Wolinsky FD. Does the relationship of the proxy to the target person affect the concordance between survey reports and Medicare claims measures of health services use? Health Serv Res. 2016:51(1):314-27.

10. Wolinsky FD, Ayres L, Jones MP, Lou Y, Wehby GL. Ullrich FA. A pilot study among older adults of the concordance between their self-reports to a health survey and spousal proxy reports on their behalf. BMC Health Serv Res. 2016;16(1):485.

11. Wolinsky FD, Jones MP, Ullrich F, Lou Y, Wehby GL. The concordance of survey reports and Medicare claims in a nationally representative longitudinal cohort of older adults. Med Care. 2014;52(5):462-8.

12. Demko CA, Victoroff $K Z$, Wotman $S$. Concordance of chart and billing data with direct observation in dental practice. Community Dent Oral Epidemiol. 2008:36(5):466-74

13. Fujita M, Sato Y, Nagashima K, Takahashi S, Hata A. Validity assessment of self-reported medication use by comparing to pharmacy insurance claims. BMJ Open. 2015;5(11):e009490.

14. Jiang L, Zhang B, Smith ML, et al. Concordance between self-reports and Medicare claims among participants in a national study of chronic disease self-management program. Front Public Health. 2015;3.

15. Kennedy AD, Leigh-Brown AP, Torgerson DJ, Campbell J, Grant A. Resource use data by patient report or hospital records: do they agree? BMC Health Serv Res. 2002;2(1):2.

16. Marks AS, Lee DW, Slezak J, Berger J, Patel H, Johnson KE. Agreement between insurance claim and self-reported hospital and emergency room utilization data among persons with diabetes. Dis Manag. 2003;6(4):199-205.

17. Palmer L, Johnston SS, Rousculp MD, Chu B-C, Nichol KL, Mahadevia PJ. Agreement between internet-based self-and proxy-reported health care resource utilization and administrative health care claims. Value Health. 2012;15(3):458-65

18. Plante C, Goudreau S, Jacques L, Tessier F. Agreement between survey data and Régie de l'assurance maladie du Québec (RAMQ) data with respect to the diagnosis of asthma and medical services use for asthma in children. Chronic diseases and injuries in Canada. 2014;34(4).

19. De-loyde KJ, Harrison JD, Durcinoska I, Shepherd HL, Solomon MJ, Young JM. Which information source is best? Concordance between patient report, clinician report and medical records of patient co-morbidity and adjuvant therapy health information. J Eval Clin Pract. 2015;21(2):339-46.

20. Glass JE, Bucholz KK. Concordance between self-reports and archival records of physician visits: a case-control study comparing individuals with and without alcohol use disorders in the community. Drug Alcohol Depend. 2011;116(1):57-63.

21. Guerard B, Omachonu V, Harvey RA, Hernandez SR, Sen B. The influence of respondent characteristics on the validity of self-reported survey responses. Health Serv Res. 2016;51(3):937-52.

22. Hunger M, Schwarzkopf L, Heier M, Peters A, Holle R. Official statistics and claims data records indicate non-response and recall bias within surveybased estimates of health care utilization in the older population. BMC Health Serv Res. 2013;13(1):1.

23. Peersman W, Pasteels I, Cambier D, De Maeseneer J, Willems S. Validity of self-reported utilization of physician services: a population study. The European Journal of Public Health. 2013;24(1):91-7.
24. Raina P, Torrance-Rynard V, Wong M, Woodward C. Agreement between self-reported and routinely collected health-care utilization data among seniors. Health Serv Res. 2002;37(3):751-74.

25. Savitz ST, Stearns SC, Zhou L, et al. A comparison of self-reported medication adherence to concordance between part $d$ claims and medication possession. Med Care. 2017:55(5):500-5.

26. Wolinsky FD, Miller TR, An H, et al. Hospital episodes and physician visits: the concordance between self-reports and medicare claims. Med Care. 2007:45(4):300.

27. Garland A, Gershengorn HB, Marrie RA, Reider N. Wilcox ME. A practical, global perspective on using administrative data to conduct intensive care unit research. Annals of the American Thoracic Society. 2015 Sep;12(9): 1373-86.

28. Tessier-Sherman B, Galusha D, Taiwo OA, Cantley L, Slade MD, Kirsche SR, Cullen MR. Further validation that claims data are a useful tool for epidemiologic research on hypertension. BMC Public Health. 2013 Dec;13(1):51.

29. Bullano MF, Kamat S, Willey VJ, Barlas S, Watson DJ, Brenneman SK. Agreement between administrative claims and the medical record in identifying patients with a diagnosis of hypertension. Med Care. 2006 May 1:486-90.

30. Zuvekas SH, Olin GL. Validating household reports of health care use in the medical expenditure panel survey. Health Serv Res. 2009;44(5p1):1679-700.

31. Costa-Santos C, Bernardes J, Ayres-de-Campos D, Costa A, Costa C. The limits of agreement and the intraclass correlation coefficient may be inconsistent in the interpretation of agreement. J Clin Epidemiol. 2011;64(3):264-9.

32. De Vet H. Observer reliability and agreement. Encyclopedia of biostatistics. 1998.

33. Bartko JJ. The intraclass correlation coefficient as a measure of reliability. Psychol Rep. 1966;19(1):3-11.

34. Nakagawa S, Schielzeth H. Repeatability for Gaussian and non-Gaussian data: a practical guide for biologists. Biol Rev. 2010;85(4):935-56.

35. Stoffel MA, Nakagawa S, Schielzeth H. rptR: repeatability estimation and variance decomposition by generalized linear mixed-effects models. Methods Ecol Evol. 2017.

36. Bolker BM, Brooks ME, Clark CJ, et al. Generalized linear mixed models: a practical guide for ecology and evolution. Trends Ecol Evol. 2009;24(3):127-35.

37. R Core Team. R: a language and environment for statistical computing. Vienna, Austria: R Foundation for Statistical Computing; 2017. URL https://www.R-project.org/

38. Yu S-T, Chang H-Y, Lin M-C, Lin Y-H. Agreement between self-reported and health insurance claims on utilization of health care: a population study. J Clin Epidemiol. 2009;62(12):1316-22.

39. Rhodes AE, Lin E, Mustard CA. Self-reported use of mental health services versus administrative records: should we care? Int J Methods Psychiatr Res. 2002;11(3):125-33.

Ready to submit your research? Choose BMC and benefit from:

- fast, convenient online submission

- thorough peer review by experienced researchers in your field

- rapid publication on acceptance

- support for research data, including large and complex data types

- gold Open Access which fosters wider collaboration and increased citations

- maximum visibility for your research: over $100 \mathrm{M}$ website views per year

At $\mathrm{BMC}$, research is always in progress.

Learn more biomedcentral.com/submissions 